\title{
Longitudinal current profile reconstruction from a wakefield response in plasmas and structures
}

\author{
R. Roussel@, ${ }^{*}$ G. Andonian, and J. B. Rosenzweig \\ Department of Physics and Astronomy, University of California, \\ Los Angeles, California 90095-1547, USA \\ S. S. Baturin 1 \\ Department of Electrical Engineering and Department of Physics, \\ Northern Illinois University, DeKalb, Illinois 60115, USA
}

(Received 7 May 2020; accepted 30 November 2020; published 17 December 2020)

\begin{abstract}
Present-day and next-generation accelerators, particularly for applications in driving wakefield-based schemes, require longitudinal beam shaping and attendant longitudinal characterization for experimental optimization. Here we present a diagnostic method which reconstructs the longitudinal beam profile at the location of the wakefield-generating source. The methods described derive the longitudinal profile of a charged particle beam solely from measurement of the time-resolved centroid energy change due to wakefield effects. As such, they are insensitive to the beam losses in post-interaction transport often found in common diagnostics. The reconstruction technique is based on a deconvolution algorithm that is fully generalizable to any analytically or numerically calculable Green's function for the wakefield excitation mechanism. This method is shown to yield precise features in the longitudinal current distribution reconstruction. We demonstrate the accuracy and efficacy of this technique using simulations and experimental examples, in both plasmas and dielectric structures, and compare them to experimentally measured longitudinal beam profiles. The limits of resolution and applicability to relevant scenarios are also examined.
\end{abstract}

DOI: 10.1103/PhysRevAccelBeams.23.121303

\section{INTRODUCTION}

Advanced acceleration techniques based on beam-driven wakefields have produced unprecedented results in terms of achievable gradients, exceeding the $\mathrm{GeV} / \mathrm{m}$ threshold in dielectrics [1], and extending up to 10 's of $\mathrm{GeV} / \mathrm{m}$ in plasmas [2]. To go beyond proof-of-concept, wakefield research now looks to optimize such acceleration schemes. In this regard, maximizing the efficiency of energy transfer to the wakefield acceleration process requires breaking the symmetry in the wake-driving beam distribution by precision manipulation of its longitudinal profile [3]. The prompt and precise characterization of the longitudinal profile is therefore critical for performance enhancement in wakefield accelerators. There are a myriad of techniques to measure the bunch profile of high brightness, picosecondscale beams, including the use of transverse deflecting

\footnotetext{
*roussel@ucla.edu
}

Published by the American Physical Society under the terms of the Creative Commons Attribution 4.0 International license. Further distribution of this work must maintain attribution to the author(s) and the published article's title, journal citation, and DOI. cavities [4], electro-optic sampling [5], and interferometry of emitted radiation [6-8].

Many diagnostic techniques incorporate beam-collimating elements in order to improve measurement resolution by restricting transverse acceptance of the beam, thus removing possible resolution degradation due to the beam's betatron size. However, such modified diagnostics may observe a distorted view of the beam distribution due to time-dependent sampling biases arising from strong correlations between the longitudinal profile and the transverse beam size. These issues are particularly prevalent in largeamplitude wakefield acceleration experiments, which, due to the presence of strong transverse forces, [9], sample a partial beam distribution at the diagnostic location. As recovery of the full beam current is needed, such effects are quite damaging to the efficacy of longitudinal beam shape diagnostics.

In order to circumvent these issues, we have developed a method to reconstruct the beam current profile at the wakefield source that relies only on the beam mean-energy measurement. The measured, time-dependent energy perturbations to the beam core, which due to the transverse uniformity of the longitudinal wakefield in the region of interest, is not affected by sample biasing due to transverse 


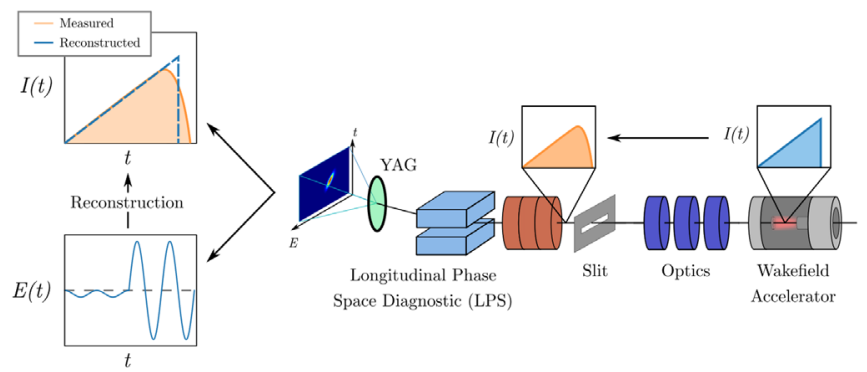

FIG. 1. Sketch of an example wakefield accelerator beamline (beam travels to the left) with a single stage wakefield accelerator and a single shot longitudinal phase space diagnostic. The reconstruction algorithm provides an accurate beam profile determination at the wakefield source, without sampling biases resulting from collimation.

beam propagation. This is in contrast with direct current measurements. The time-resolved mean energy is acquired with a commonly employed single-shot longitudinal phase space (LPS) diagnostic, consisting of a deflecting cavity and dipole magnet spectrometer [10]. This measurement, along with knowledge of the single particle wakefield inside the structure or plasma, allows one to create a high-fidelity reconstruction of the bunch profile at the wakefield source, as seen in Fig. 1. This measurement of the energy centroid is differential (made via a comparison between the time dependent mean energy when the wakefield source is present or not), so in the absence of significant longitudinal motion within the distribution it accounts for downstream optics and external sources of energy change. This enables acquisition of the bunch profile at the wakefield source in cases where transverse beam optics constraints (i.e., apertures) prevent accurate, direct current profile measurements.

Further, and most strikingly, the application of the reconstruction method yields profiles with a high degree of precision and even subresolution features beyond the diagnostic threshold, particularly when the wake functions of the source are well-understood. In the examples we employ to demonstrate these methods, we show that when these reconstructed profiles are utilized, the predicted wakefield response is accurately corroborated by the measured effects in the drive and witness bunches, resulting in a comprehensive and self-consistent description of the wakefield interaction. Even in cases where the measured current profile is resolvable with other methods, our reconstruction technique provides a detailed cross-check that can yield further physical insight into the interaction, without the need to introduce extra diagnostic instrumentation.

The reconstruction method is employable over a wide range of conceivable wakefield interaction scenarios. Beyond acceleration, beam-driven wakefields are already used for phase space manipulations, such as beam compression [11], linear ramp shaping [12] or dechirping [13-15]. The information gleaned from the manipulating media can doubly yield additional detailed bunch profile information by application of the signal reconstruction method presented here.

\section{BACKGROUND}

The longitudinal profile reconstruction technique discussed here is based on a signal deconvolution of time-resolved energy change measurements due to the wakefield. For a longitudinal wakefield, the drive beam initially experiences deceleration specific to the nature of the modes supported by the medium (dielectric, corrugated structure, or plasma). This manifests itself as a timedependant change in beam energy, $\Delta U$, which is proportional to the induced wake potential, $W(s)$, which is defined as the convolution of the longitudinal beam distribution and the wake function,

$$
W(s)=\int_{0}^{s} \rho_{z}(\tilde{s}) G_{z}(s-\tilde{s}) d \tilde{s},
$$

where $\rho_{z}(s)$ is the longitudinal beam distribution, and $G_{z}(s)$ is the Green's function representing the longitudinal wake function, or longitudinal wake potential of a point particle [16]. Equation (1) is valid under the assumption of a pencil-like beam, where the transverse RMS beam size $\sigma_{r}$ is small compared to either the plasma wavelength or vacuum channel size, in the case of a wakefield (e.g., dielectric or metallic) structure. With known functions $W(s)$ and $G_{z}(s)$, a practical method of solving for $\rho_{z}(s)$ in this integral equation involves the application of Laplace transformations.

We define the forward and inverse Laplace transformations as $[17,18]$

$$
\mathcal{L}[f(s)]=\int_{0}^{\infty} f(s) e^{-p s} d s
$$

and

$$
\mathcal{L}^{-1}[F(p)]=\frac{1}{2 \pi i} \lim _{R \rightarrow \infty} \int_{\kappa-i R}^{\kappa+i R} e^{s p} F(p) d p,
$$

where $\kappa$ is a real number such that the line $p=\kappa$ in the complex plane avoids singularities of $F(p)$. According to the convolution theorem for the Laplace transformation [17], the Laplace image of the convolution integral with the variable upper limit is equal to the product of the Laplace images of the functions under the integral

$$
\mathcal{L}\left[\rho_{z} * G_{z}\right]=\mathcal{L}\left[\rho_{z}(s)\right] \mathcal{L}\left[G_{z}(s)\right] .
$$

Here $*$ refers to a convolution, as shown in Eq. (1) $\rho_{z} * G_{z} \equiv \int_{0}^{s} \rho_{z}(\tilde{s}) G_{z}(s-\tilde{s}) d \tilde{s}$. After rearrangement, the charge density, $\rho_{z}(s)$, can be solved for by applying an inverse Laplace transformation on the quotient, as 


$$
\rho_{z}(s)=\mathcal{L}^{-1}\left[\frac{\mathcal{L}(W)}{\mathcal{L}\left(G_{z}\right)}\right] .
$$

In practice, the wake potential, proportional to the timeresolved energy change, is measurable with high precision, and the Green's function is analytically calculable for many types of media.

In the generalized case when the Green's function $G_{z}(s)$ is given numerically, as are the measured values of $W(s)$, one can solve Eq. (1) approximately by replacing the convolution $\rho_{z} * G_{z}$ with the quadrature formula on a given mesh $s_{n}=n h$, with the mesh step $h$. The problem of Eq. (1) in this case is reduced to a linear system

$$
W\left(s_{n}\right)=\sum_{j=m}^{n} A_{m} \rho_{z}\left(s_{m}\right) G_{z}\left(s_{n}-s_{m}\right)
$$

that should be solved for $\rho_{z}\left(s_{m}\right)$. Here, $A_{m}$ are coefficients of the chosen quadrature formula. At this point, Eq. (5) and Eq. (6) are completely general, and the details of the beam reconstruction are dependent on the specific Green's function.

Hence, a longitudinal beam diagnostic method can be devised by selection of the medium with a well-known longitudinal wake response and measurement of the beam energy change due to the self-induced wakefield. In many applications, such as wakefield acceleration or dechirping, the manipulating medium already provides the necessary change in beam energy to make the concept viable. It is also notable that this method provides an independent measure of beam charge, for proper normalization of $G_{z}(s)$, with the integral $\int \rho_{z}(s) d s=Q$, where $Q$ is the total charge.

In the following sections of this work, we explore the implications of this technique with specific application to reconstructing the bunch profile using different examples. First, we examine the simple case of a single mode wake function, which can be used in certain (linear and quasinonlinear) cases of plasma wakefield acceleration [19] and a limited scenario in dielectric wakefield acceleration. Then we extend the analysis to a more general approach that incorporates multiple modes, as well as arbitrary Green's functions, which are essential for cases where the singlemode description cannot capture the complete wakefield response. Experimentally derived examples of reconstructed beam profiles are presented using time-resolved energy measurements from plasma and dielectric wakefield acceleration experiments at the Argonne Wakefield Accelerator [20]. Further, we examine the fidelity of the diagnostic scheme under different situations and in the presence of measurement uncertainties.

\section{SINGLE MODE DESCRIPTION}

In the first case, we consider a single mode Green's function

$$
G_{z}(s)=G_{0} \cos \left(k_{0} s\right),
$$

where $k_{0}$ is the wave number associated with the fundamental mode. We then apply Eq. (5) to derive the longitudinal profile $\rho_{z}(s)$. Consideration of this simple wake function is relevant for cases such as a dielectric wakefield structure that can be optimized for a single-mode operation [21,22], or quasilinear plasma wakefields [19].

The Laplace image of Eq. (7) is

$$
\mathcal{L}\left[G_{0} \cos \left(k_{0} s\right)\right]=G_{0} \frac{p}{p^{2}+k^{2}} .
$$

The longitudinal profile is then determinable from the inverse Laplace transformation as prescribed by Eq. (5)

$$
\rho_{z}(s)=\mathcal{L}^{-1}\left[\bar{W}(p) \frac{k_{0}^{2}+p^{2}}{G_{0} p}\right]
$$

where $\bar{W}(p) \equiv \mathcal{L}[W(s)]$. By using the linearity of the Laplace transformation, we can expand Eq. (9) to obtain

$$
\rho_{z}(s)=\frac{1}{G_{0}} \mathcal{L}^{-1}[p \bar{W}(p)]+\frac{k_{0}^{2}}{G_{0}} \mathcal{L}^{-1}\left[\frac{\bar{W}(p)}{p}\right] .
$$

We notice that

$$
\mathcal{L}\left[W^{\prime}(s)\right]=p \bar{W}(p)-W(0)
$$

and

$$
\mathcal{L}\left[\int_{0}^{s} W(\tilde{s}) d \tilde{s}\right]=\frac{\bar{W}(p)}{p} .
$$

Here prime in $W^{\prime}(s)$ denotes the total derivative.

Without loss of generality we chose the origin in $s$ such that $W(0)=0$. Finally, with Eq. (11) and Eq. (12), Eq. (10) can be written as

$$
\rho_{z}(s)=\frac{W^{\prime}(s)}{G_{0}}+\frac{k_{0}^{2}}{G_{0}} \int_{0}^{s} W(\tilde{s}) d \tilde{s} .
$$

As a check, in the simple case of constant $W(s)$, Eq. (13) represents a beam current density distribution that increases linearly, accompanied by a short current spike at the head of the beam. This ramped beam is a familiar scenario found in theoretical treatments of beam-driven wakefield acceleration (e.g., [3,23-27]).

\section{A. Example: Plasma wakefield acceleration}

As an example of the reconstruction method's applicability we apply it in the context of longitudinally ramped bunches, produced by emittance exchange (EEX) [28], interacting with a plasma wakefield accelerator to obtain a high transformer ratio, as described in Ref. [19]. In this case, several factors unique to this experiment (discussed later on) prevent acquiring an accurate measurement of the beam profile. 


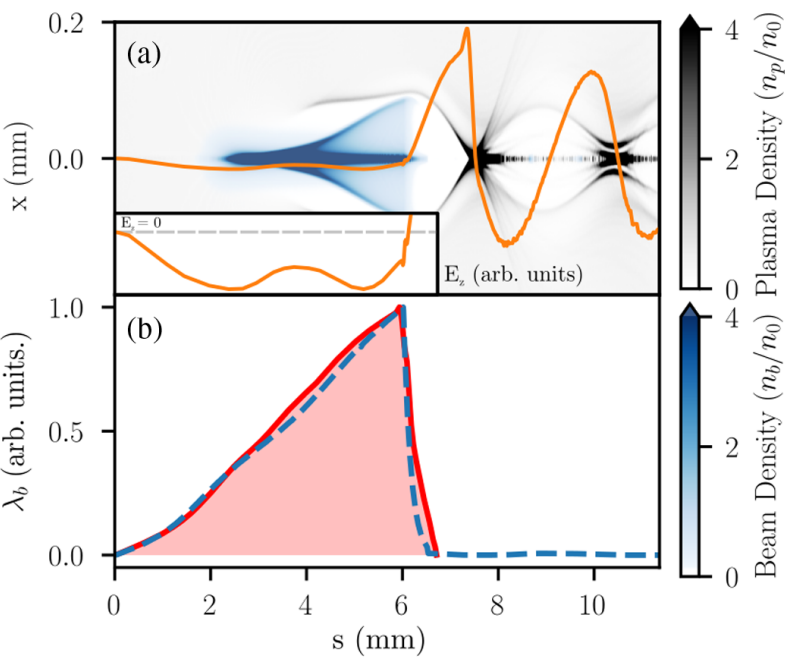

FIG. 2. WARP simulation of a ramped beam after propagating $34 \mathrm{~mm}$ through a uniform plasma. (a) Longitudinal cross-section of the beam (blue) and plasma (grey) electron densities with the on-axis longitudinal electric field. Inset: magnified view of the on-axis longitudinal wakefield. (b) Comparison between PIC simulated longitudinal current distribution (blue, dashed) and the single mode bunch reconstruction (red, filled) from the simulated wakefield $E_{z}$.

In order to apply the reconstruction, we first consider that the experimental conditions give rise to a regime where the wakefields within the bunch are described by linear plasma wakefield theory, where the beam density $n_{b}$ is much smaller than the plasma density $n_{p}$. The perturbation to the plasma density is given by $[29,30]$.

$$
\frac{\partial^{2} n_{1}(s)}{\partial^{2} s^{2}}+k_{p}^{2} n_{1}(s)=-k_{p}^{2} n_{b}(s)
$$

In this experiment, the beam is much narrower than the plasma skin-depth $k_{p}^{-1}=c / \omega_{p}$, and so the longitudinal wakefield is nearly constant across the radial extent of the beam. Furthermore, the ramped beam profile causes a transition to the quasi-nonlinear regime, so the radial dependence of the longitudinal wake disappears completely. Thus the representative Green's function is given by the familiar form seen in Eq. (7) where $k_{0}=k_{p}$ is the characteristic plasma wave number. By using $k_{p}$ calculated from a given plasma density, and the experimental measurement of the time dependent energy gain as a proxy for the average wakefield in the plasma, we reconstruct the longitudinal beam profile inside the plasma using Eq. (13).
Some comments on the validity of this method as applied to the "quasi-nonlinear" case are warranted. A fully nonlinear plasma blowout is due to short $\left(k_{p} \sigma_{z}<1\right)$, high charge beams [31]. However, the experiments reported in Ref. [19] used a long triangularly shaped beam to reach blowout conditions through an extended build-up of beam charge density over several plasma wavelengths. In this case, the maximum beam charge density is larger than the unperturbed plasma density. With this strong perturbation, electrons are expelled from the beam channel, creating a rarefied "bubble" region around the beam where no plasma electrons are present. However, the transverse motion of the plasma electrons is nonrelativistic due to the adiabatic excitation method, and the maximum bubble radius, $r_{m}$, is small compared to the plasma skin-depth. As a result, the longitudinal near-axis wakefield inside the drive bunch is dominated by fields generated by the population of plasma electrons reacting to the effectively linear perturbation to the plasma density outside the bubble [32]. Thus the longitudinal wakefield response can be characterized as nearly linear, and can be approximated by a single mode Green's function, of the same longitudinal dependence as the linear regime limit.

This effect is demonstrated in Fig. 2 through the use of the particle-in-cell code WARP [33]. In the simulation, a linearly ramped beam with parameters matching those in Ref. [19], and displayed in Table I, was injected into a uniform plasma with a density $n_{0}=1.3 \times 10^{14} \mathrm{~cm}^{-3}\left(\lambda_{p}=3 \mathrm{~mm}\right)$. A longitudinal slice of the simulation is shown in Fig. 2(a) along with the on-axis longitudinal wakefield. Inside the drive beam, the wakefield is linear in nature as evidenced by its sinusoidal dependence on $s$ (see Fig. 2(a) inset). In Fig. 2(b), the single mode reconstruction [Eq. (13)] technique is used to reconstruct the beam profile from the simulated wakefield. The reconstructed bunch profile shows excellent agreement with the simulated bunch profile, including reproduction of fine features such as the curvature at the beam head due to defocusing forces experienced by the beam before the bubble region fully develops.

It is important to stress that the reconstruction using a single mode Green's function is only accurate within the drive beam for these experiments. In the beam region, the wakefield contribution is dominated by the quasilinear plasma perturbation outside the bubble region. After the drive electron density suddenly drops to zero (in a distance $<k_{p}^{-1}$ ), the bubble collapses with an attendant nonlinear response, and the wakefield is no longer approximated by a single, linear mode response.

TABLE I. Parameter for the WARP simulation and experiment from the Ref. [19].

\begin{tabular}{lccccc}
\hline \hline Beam energy & Charge & Beam length & Beam spot size & Plasma density & Plasma length \\
\hline $40 \mathrm{MeV}$ & $1.8 \mathrm{nC}$ & $6 \mathrm{~mm}$ & $200 \mu \mathrm{m}$ & $1.3 \times 10^{14} \mathrm{~cm}^{-3}$ & $8 \mathrm{~cm}$ \\
\hline \hline
\end{tabular}




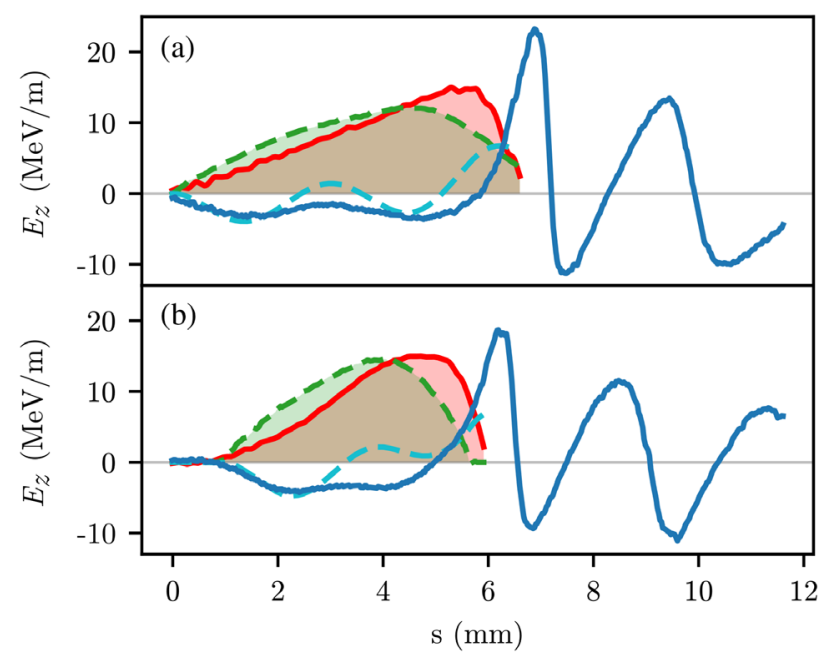

FIG. 3. Wakefield measurement (blue), normalized current reconstruction (red), normalized current profile measurement (green, dashed), and wakefield prediction from current measurement (light blue, dashed) from single shot longitudinal phase space measurements of the beam after a plasma accelerator. Measurements from two different beam shapes (a) linear ramp, (b) linear ramp with parabolic head are shown.

Results from the use of our reconstruction method for the quasi-nonlinear experimental case described by [19] are plotted in Fig. 3. In this particular experiment at the Argonne Wakefield Accelerator, an emittance exchange beamline [28] was used to shape the temporal profile of the beam into two different ramped profiles, one without any leading perturbation and one with a parabolic head perturbation. Here, the time-dependent energy change of the drive and witness bunches was measured along with the longitudinal current distribution using a single-shot longitudinal phase space diagnostic (Fig. 1) [10]. A 100 micron wide horizontal slit, placed immediately upstream of the diagnostic was used to improve the temporal resolution of the measurement.

The reconstructed bunch profile (shown in red) is created by following the steps listed here. The time-dependent wakefield was extracted from the observed beam energy change due to the wakefield interaction averaged over the $8 \mathrm{~cm}$ long plasma column [34]. The plasma wave number, $k_{p}$, is calculated by measuring the distance between adjacent minima in the wakefield in the drive region, which corresponds well to the plasma wavelength $2 \pi / k_{p}$. We apply the reconstruction method [Eq. (13)] to the region before the first wakefield maximum, where simulations indicate that the beam experiences the quasinonlinear regime. In the first case, Fig. 3(a) the reconstruction shows a bunch with a near-linear ramp over roughly two plasma periods along with a short tail. The development of a tail is consistent with beamline dynamics simulations as a symptom of the emittance exchange shaping process and strong focusing before the plasma stage [35]. In Fig. 3(b) we observe that the reconstructed beam profile has a linear ramp with a parabolic head perturbation. This perturbation has been shown analytically to flatten the decelerating wakefield inside the drive [24] when the perturbation stretches over a plasma wavelength, which is consistent with our observations of the wakefield itself.

The predicted single mode wakefield from the measured current distribution is also plotted in Fig. 3. This was calculated by convolving the measured current profile with the single mode Green's function Eq. (7) where $k_{0}$ is derived from the measure plasma wavelength and the amplitude $G_{0}$ is set to match the observed maximum decelerating wakefield.

The experimentally measured current profiles show significant differences when compared to the reconstructed profiles, demonstrating the sampling biases when collimation is used, which is an expected result due to three factors. First, as a consequence of the emittance exchange bunch shaping process, longitudinal slices of the beam have varying vertical profiles, where the bunch slice that contains the highest current is also the largest transversely [35]. Second, the presence of strong transverse forces in the multiperiod plasma wakefield response, results in nonuniform focusing along the drive bunch length, which when propagated through the diagnostic beamline, leads to further longitudinal-transverse correlations. As a result of these factors, when using horizontal collimation to improve the temporal resolution, we sample only a small portion of the vertical beam distribution, which strongly biases the faction of charge collected from each temporal slice. Finally, significant sources of emittance growth before the diagnostic exist, both inside the plasma as a result of density mismatch [36] and multiple scattering [37] in beryllium windows used to separate the plasma from the rest of the beamline. This accentuates any differences in vertical beam slice size relative to the collimator dimensions. These factors explain why the measured, normalized, current profiles shown in Fig. 3 show greater relative current density in the beam head and smaller current density in the tail, when compared to the reconstruction. Furthermore, the predicted wakefield from the measured current distribution clearly does not match the observed wakefield, casting further doubt on the accuracy of the measured current profile. The reconstruction method relies only on the differential centroid energy measurement, thus providing a profile characterization free of these artifacts and is consistent with the observed wakefield.

\section{MULTIMODE DESCRIPTION}

The single-mode reconstruction technique works for a specific subset of problems, and is extremely useful for a rapid profile determination. However, for cases where the Green's function of the wakefield device is precisely known, either analytically or through simulation, a general approach can be used to expose finer features in the beam 
profile. This includes both dielectric [38] and corrugated metal structures as well as other media where the modal description is calculated numerically, such as the woodpile structure considered recently [22], where the wake function of a single particle is describable by a series of modes. In this section, we extend the applicability of the reconstruction algorithm beyond the single-mode description of the previous section.

\section{A. Multimode Green's function: Few-mode case}

When extending the analysis to a multimode structure, the Green's function of Eq. (7) can be replaced by a superposition of different mode contributions

$$
G_{z}(s)=\sum_{n=0}^{N} C_{n} \cos \left(k_{n} s\right)
$$

First, we calculate the Laplace image of the Green's function using the linearity of the Laplace transformation

$$
\bar{G}(p)=\mathcal{L}\left[\sum_{n=0}^{N} C_{n} \cos \left(k_{n} s\right)\right]=\sum_{n=0}^{N} \frac{C_{n} p}{k_{n}^{2}+p^{2}} .
$$

To find $\rho_{z}(s)$ according to the Eq. (5) and the definition of the inverse Laplace transformation Eq. (3) the integral

$\rho_{z}(s)=\frac{1}{2 \pi i} \int_{\kappa-i \infty}^{\kappa+i \infty} \frac{\bar{W}(p) e^{s p} \prod_{n=0}^{N}\left(k_{n}^{2}+p^{2}\right)}{p \sum_{n=0}^{N}\left\{C_{n} \prod_{m \neq n}\left(k_{m}^{2}+p^{2}\right)\right\}} d p$

needs to be evaluated. An analysis of the integrand unveils an essential singularity at the points $p= \pm i \infty$ as well as poles that correspond to the zeros of the polynomial in the denominator:

$$
p \sum_{n=0}^{N}\left\{C_{n} \prod_{m \neq n}\left(k_{m}^{2}+p^{2}\right)\right\}=0 .
$$

The detailed evaluation of this integral is given in Appendix.

Again taking the origin in $s$ such that $W(0)=0$ we arrive at the final expression for the charge density

$$
\begin{aligned}
\rho_{z}(s)= & \frac{W^{\prime}(s)}{\sum_{n=0}^{N} C_{n}}+\frac{\prod_{n=0}^{N} k_{n}^{2}}{\sum_{n=0}^{N}\left\{C_{n} \prod_{m \neq n} k_{m}^{2}\right\}} \int_{0}^{s} W(\tilde{s}) d \tilde{s} \\
& +\int_{0}^{s} K(s-\tilde{s}) W(\tilde{s}) d \tilde{s} .
\end{aligned}
$$

with the kernel $K(s)$ given by

$$
K(s)=\sum_{p_{i}} \operatorname{Res}\left[\frac{e^{s p} \prod_{n=0}^{N}\left(k_{n}^{2}+p^{2}\right)}{p \sum_{n=0}^{N}\left\{C_{n} \prod_{m \neq n}\left(k_{m}^{2}+p^{2}\right)\right\}}\right] .
$$

Here $p_{i}$ are the roots of the Eq. (18) that are enclosed by the integration contour (see Appendix) except for the root at $p=0$. For the case of two and three modes, the residues in
Eq. (20) can be evaluated analytically, as the equations for the poles Eq. (18) are quadratic and bi-quadratic equations respectively. It is still possible to evaluate the four-mode case but this involves Cardano formulas for the cubic equation. If the number of modes is greater then four, one should proceed with a numerical solution of Eq. (18) and substitution of the calculated zeros into the residue formula in Eq. (20).

\section{B. Arbitrary Green's function}

The evaluation of Eq. (1) by a Laplace transformation is preferable as the integrals are calculated exactly, thus removing any error due to approximations. However, in some cases when the Green's function is computed combining numerical simulation and extrapolation approach (see Ref. [39]), it may be more convenient to consider a numerical reconstruction scheme. In this case the Green's function and the wake potential $W(s)$ are given on a mesh $s_{n}=n h$ where we assume that the mesh $s_{n}$ is uniform, with step size $h$. We rewrite Eq. (1) on this mesh as

$$
W\left(s_{n}\right)=\int_{0}^{s_{n}} \rho_{z}(\tilde{s}) G_{z}\left(s_{n}-\tilde{s}\right) d \tilde{s} .
$$

If we assume that $h \ll 1$ then the integral on the right-hand side can be approximated using the quadrature formula. If we chose the mesh for the integral $s_{m}=m h$ (the same as the initial mesh $s_{n}$ ) then we write the integral as

$$
\int_{0}^{s_{n}} \rho_{z}(\tilde{s}) G_{z}\left(s_{n}-\tilde{s}\right) d \tilde{s} \approx \sum_{m=1}^{n} Q_{m} \rho_{m} G_{n, m} .
$$

Here $Q_{m}$ are coefficients for the corresponding quadrature formula, $G_{n, m}=G_{z}\left(s_{n}-\tilde{s}_{m}\right)$ is the element of the square matrix G, and $\rho_{m}=\rho_{z}\left(\tilde{s}_{m}\right)$ is the element of the unknown vector $\rho$. Introducing the notation $W\left(s_{n}\right) \equiv W_{n}$ and using Eq. (22), Eq. (21) becomes a square system of linear equations of the form

$$
W_{n}=\sum_{m=1}^{n} Q_{m} \rho_{m} G_{n, m} .
$$

The most appropriate quadrature formula for the equidistant mesh and the case here is the trapezoidal rule with coefficients $Q_{m}$ given by

$$
Q_{m}= \begin{cases}1 / 2 & m=1, n \\ 1 & m \neq 1, m \neq n .\end{cases}
$$

The matrix $G_{n, m}$ has a triangular form, so all values of $\rho_{m}$ can be calculated using a simple recurrent formula that with Eq. (24) for $k>2$ reads 
TABLE II. Experimental parameter of Ref. [20].

\begin{tabular}{lcccccc}
\hline \hline Beam energy & Charge & Vacuum gap & Slab thickness & Width & Length & $\varepsilon$ \\
\hline $48 \mathrm{MeV}$ & $\sim 2 \mathrm{nC}$ & $2.5 \mathrm{~mm}$ & $150 \mu \mathrm{m}$ & $1.27 \mathrm{~cm}$ & $15 \mathrm{~cm}$ & 3.75 \\
\hline \hline
\end{tabular}

$$
\rho_{k}=2 \frac{W_{k}-W_{1}-\frac{h}{2} \rho_{1} G_{k, 1}-h \sum_{l=2}^{k-1} \rho_{l} G_{k, l}}{h G_{k, k}} .
$$

To initiate the calculations, we again assume $W(0)=0$ as well as $\rho_{z}(0)=0$. This allows us to calculate $\rho_{1}$ as

$$
\rho_{1}=\frac{2 W_{1}}{h G_{1,1}}
$$

and $\rho_{2}$ as

$$
\rho_{2}=2 \frac{W_{2}-W_{1}-\frac{h}{2} \rho_{1} G_{2,1}}{h G_{2,2}} .
$$

If we assume that the total number of mesh points is $N$ and $\rho_{0}=\rho_{z}(0)=0$ then the total charge can be calculated using the same trapezoidal rule as follows

$$
Q=\frac{h \rho_{N}}{2}+h \sum_{m=1}^{N-1} \rho_{m} .
$$

\section{Example: Dielectric wakefield acceleration}

As a relevant example for the multimode case we consider the dielectric wakefield acceleration experiment described in Ref. [20]. Similar to the plasma example, this experiment used a linearly ramped bunch, generated using the emittance exchange beam line [28] and sent through a dielectric structure with planar symmetry. A single shot wakefield measurement system [10] was utilized to map out the wakefield behind this triangular shaped driver. Parameters of the dielectric slabs as well as external experimental conditions of Ref. [20] are summarized in Table II.

For a rectangular structure, the Green's function is represented by a double sum over $x$ and $y$ modes as

$$
G_{z}^{I}(s)=\sum_{n_{1}}^{N_{x}} \sum_{m=1}^{N_{y}} A_{n, m} \cos \left(k_{n, m} s\right)
$$

(see Refs. [40-42]). In this specific case, to achieve high accuracy $(<0.1 \%)$ the Green's function has to include $N_{x}=80 x$-modes and $N_{y}=50 y$-modes, for a total of 4000 modes. First, we apply Eq. (19) to the experimentally measured wakefield inside the bunch. We then apply a lowpass filter to reduce numerical noise that comes from the derivative term. The power spectrum of the distribution that resulted from the reconstruction is simply zeroed above a certain cutoff frequency that is considered as a filter parameter. The filter parameter was adjusted manually until the difference between convolution and measurement did not exceed $15 \%$, and most numerical artifacts were eliminated. The bunch profile that resulted from the reconstruction performed in this manner is presented in Fig. 4(b) and compared to the charge density measured with the LPS diagnostic from Ref. [20], Fig. 4(a). The reconstruction provides significant new insights into the fine features of the charge density profile as well as clarifying adjustments to the bunch length and the shape of the tail.

Next, the Green's function was adjusted to account for the effects of the group velocity $v_{g}$ as $G_{z}(s)=G_{z}^{I}(s)$ $\left[1-s / L\left(1-v_{g}\right)^{-1}\right]$. For the modes that give dominant contribution to the wakefield, $v_{g} \approx 0.8 c$ on average. The attenuated Green's function was evaluated numerically [Fig. 5(a)] and then Eq. (25) with Eq. (26) and Eq. (27) were applied to restore the charge density profile. As before, numerical noise that comes from the discretization of the convolution integral was filtered out using low-pass filter. Filter parameters were optimized based on the same strategy as before, namely the reconstructed distribution was convoluted with the Green's function and compared with the initial measured wakefield inside the bunch; the

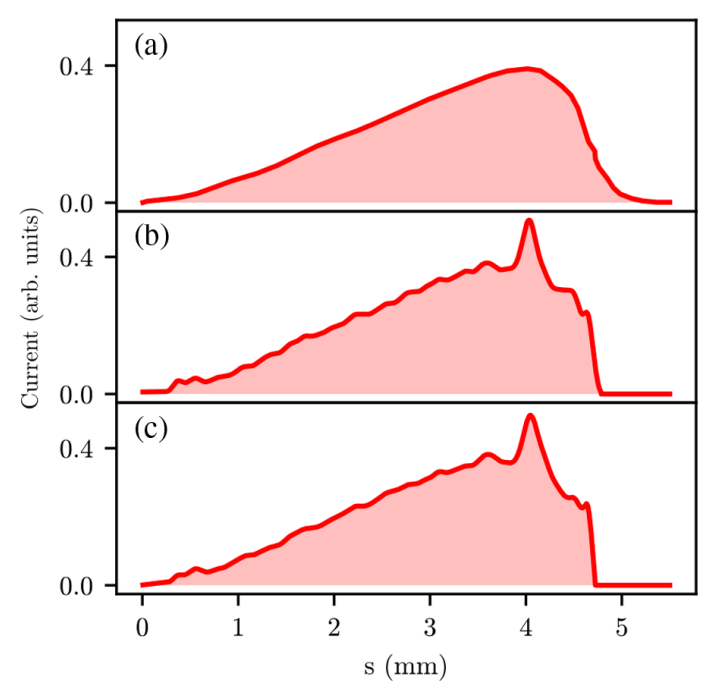

FIG. 4. (a) Normalized charge density from Ref. [20] measured with the LPS diagnostic. (b) Normalized charge density at the wake field accelerating structure reconstructed using Eq. (19) (c) Normalized charge density at the wake field accelerating structure reconstructed using Eq. (25). 


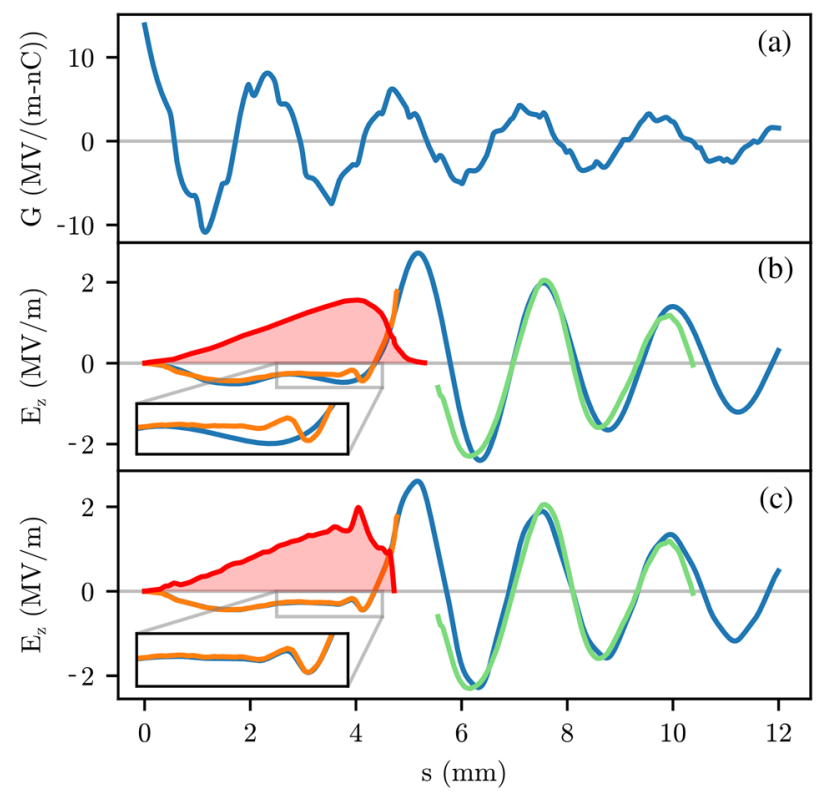

FIG. 5. (a) Green's function for the structure that was used in Ref. [20] for measuring the wake field. (b) Charge density as measured via LPS diagnostics (red, filled), measured wake field inside the driver (orange), measured wake field behind the driver (green) and theoretical calculation of the full wake field based on a Green's function and charge density measure via LPS diagnostics (blue) (c) Reconstructed charge density (red, filled), measured wake field inside the driver (orange), measured wake field behind the driver (green) and theoretical calculation of the full wake field based on a Green's function and reconstructed charge density (blue).

filter parameter was then adjusted to reduce error between the convolution and the measurement. The largest errors occur at the bunch tail, however $15 \%$ accuracy is enough to remove the major part of the numerical noise while resolving the spike in the charge density closest to the tail. Further smoothing of the distribution and elimination of this spike results in a quite large $40 \%-60 \%$ difference between the measured value and the reverse convolution of the wake at these locations.

We also use the reconstruction technique to evaluate the total charge by applying Eq. (28). We calculate the total charge for the reconstructed profile to be $Q=1.8 \mathrm{nC}$. This number is in agreement with the measured charge of $\sim 2 \mathrm{nC}$ within the experimental errors, yielding an independent method to corroborate charge measurements.

It is important to note that profiles which are reconstructed using the two different approaches [Fig. 4(b) and Fig. 4(c)] match quite well. Slight differences at the tail of the distribution may be explained by slight differences in the Green's functions, as well as variation of the parameters of the low pass filter.

In Fig. 5(c) we compare the theoretical prediction of the wakefield generated by a reconstructed bunch with the measurements of the Ref. [20]. To produce the theoretical wakefield [blue line in Fig. 5(c)] the Green's function for this structure Fig. 5(a) is numerically convoluted with the reconstructed bunch profile [Fig. 4(b)]. As expected, the measured wakefield inside the bunch [orange line in Fig. 5 (c)] coincides with the theoretical prediction. This indicates that the reconstruction procedure has been successful and the bunch profile agrees with the measured wakefield. Furthermore, we observe that behind the bunch the calculated wakefield is in very close agreement with experimental observations [green line in Fig. 5(c)] from Ref. [20], providing further validation of this technique.

We note that in comparison to the LPS diagnostics [Fig. 5(b)] reconstructed charge density better reproduces measured wakefield inside the bunch. The observed difference between the LPS diagnostic measurement Fig. 5(b) and the reconstructed profile Fig. 5(c) comes from the linearly ramped beam, which has a strong vertical-longitudinal correlation between particles in the tail. These particles are excised due to the aperture in the LPS diagnostic, thus leading to a bunch shape that is not well represented at the tail end.

It should be noted as well that the long-range wakefield is consistent with the measurements in both cases, as higher order harmonics in the long-range wakefield that couple to the fine features of the bunch longitudinal profile are heavily suppressed by the bunch length.

\section{DISCUSSION}

It is apparent from comparisons between the experimental and simulated wakefields that the reconstruction method accurately predicts the drive beam profile present during the wakefield interaction. Several practical issues must carefully be taken into account when applying these reconstruction algorithms in order to reduce error. The accuracy of the reconstruction relies not only on the measurement accuracy of the wakefield, but also on the physical validity of the Green's function used to describe the wakefield response. For example, in the plasma wakefield case, the plasma wave number was estimated by measuring the separation between adjacent wakefield minima in the drive beam (as well as from independent measurements of the plasma density). Variations of the parameter $k_{p}$ in this case, changes the weighting between the derivative and integration terms in the Eq. (13), leading to greater variations in the tail of the reconstructed beam, where the integration term is typically dominant.

It is also important to consider that the single-mode reconstruction validity [Eq. (13)] is extendable to certain multimode cases as well. By inspecting Eq. (19) we conclude that if $\max |K(s)|$ given by Eq. (20) is much smaller than the multiplier in front of the integral in Eq. (19) one may neglect the third convolution term and the reconstruction formula resembles Eq. (13), except for multipliers in front of the integral and derivative. Interestingly these factors could be found by a simple optimization procedure with an ansatz, 


$$
\rho_{z}(s)=A\left(W^{\prime}(s)+B \int_{0}^{s} W(\tilde{s}) d \tilde{s}\right)
$$

which is convoluted with the known Green's function. Then, the constants $A$ and $B$ are varied to minimize

$$
\max \left|W(s)-\int_{0}^{s} \rho_{z}(\tilde{s}) G_{z}(s-\tilde{s}) d \tilde{s}\right| .
$$

The smoothing strategy for the low-pass filter that was applied in the example cases relies on a similar optimization method. The back-convolution of the filtered result is compared to the measured wakefield and the maximum modulus of the difference is kept below a certain threshold. This strategy shows the trade-off between smoothness of the reconstructed profile and accuracy of the reconstruction procedure.

The universal solution introduced in Sec. IV B has a built in error that is connected with the discretization in Eq. (6) of the convolution integral Eq. (1). Oversampling of the measured data for the $W(s)$ and subsequent filtering solves this problem, however analytical evaluation of Eq. (5) is preferable, as it does not produce additional errors and requires less aggressive filtering. This in turn enables finer resolution in certain scenarios.

The proposed method can be directly applied to structures with different transverse geometries using their respective Green's functions, given a pencil-like beam approximation is applicable. If the transverse size of the beam is significant (close to the size of the vacuum gap of the structure or plasma skin-depth) then additional considerations regarding the transverse form-factor must be incorporated into the reconstruction procedure. This is critical for some linear PWFA experiments (as might be encountered at AWAKE [43], for example). It is also especially important in the multimode case, as modal amplitudes have to be modified to account for the transverse beam shape and size. These considerations can be taken into account in a straightforward manner, using a computational approach.

\section{SUMMARY}

In this work, we have suggested an innovative approach in the experimental methodology of determining the longitudinal charge distribution, based on deconvolution of measured longitudinal wakefield effects in a beam. The algorithm is generalizable to a wide variety of experimental scenarios and as such, is applicable to a variety of wakefieldbased media (plasma, dielectric, corrugated, photonic and Bragg structures etc.). It permits, in these applications, reconstruction of the bunch profile with high-precision, reproducing fine features that may not be observable with other beam measurement methods. This technique has been successfully applied to reconstruct longitudinal charge distribution in the plasma wakefield acceleration experiment described in Ref. [19], and in the dielectric wakefield acceleration experiment described in Ref. [20]. It is important to emphasize that the methods presented, whether they are single- or multi-mode, yield excellent results for the asymmetric beam shapes of highest interest. Enhanced beam features with finer resolution may require the multimode approach, while the universal solution proposed here has some inherent numerical fluctuations that must be filtered to obtain robust results. Regardless, our method does not suffer from biasing due to transverse subsampling of the beam with collimators.

One may examine the future application of this method in next-generation PWFA experiments, which are reliant on high-resolution temporal diagnostics [44]. Generally, PWFA experiments have relatively long interaction lengths, and associated energy loss, and thus a large effective projected emittance growth due to betatron oscillation phase-mixing. In this case there would be significant challenges in transporting the entire beam to the diagnostics. For highly resolved time measurements, which depend on the slice emittance, our energy-based reconstruction technique is able to produce resolution on the scale of $\sim \mathrm{fs}$, and is applicable in both short pulse and long-ramped pulse modes.

The highly flexible profile reconstruction method is applicable to advanced accelerators based on wakefields, conventional accelerators, machine-learning optimization routines, and for compact diagnostics designed to probe bunch profiles at any point along an accelerator.

\section{ACKNOWLEDGMENTS}

We would like to thank Q. Gao for providing the data from [20] for analysis, and the Argonne Wakefield Accelerator staff for fruitful discussions. This work is supported by the Department of Energy, Office of High Energy Physics, under Grants No. DE-SC0017648 and No. DE-SC0009914.

\section{APPENDIX: EVALUATION OF THE INTEGRAL IN EQ. (17)}

In this appendix we present a detailed evaluation of the inverse Laplace transformation for the multimode case. For convenience we reproduce the inverse Laplace integral Eq. (17):

$\rho_{z}(s)=\frac{1}{2 \pi i} \int_{\kappa-i \infty}^{\kappa+i \infty} \frac{\bar{W}(p) e^{s p} \prod_{n=0}^{N}\left(k_{n}^{2}-p^{2}\right)}{p \sum_{n=0}^{N}\left\{C_{n} \prod_{m \neq n}\left(k_{m}^{2}+p^{2}\right)\right\}} d p$.

In the limit of $p \rightarrow \pm i \infty$, the products in the numerator, as well as the product in the denominator, in the expression under the integral could be modified as 


$$
\begin{aligned}
& \prod_{n=0}^{N}\left(k_{n}^{2}+p^{2}\right) \underset{p \rightarrow \pm i \infty}{\longrightarrow} p^{2 N}, \\
& p \sum_{n=0}^{N}\left\{C_{n} \prod_{m \neq n}\left(k_{m}^{2}+p^{2}\right)\right\} \underset{p \rightarrow \pm i \infty}{\longrightarrow} p^{2 N-1} \sum_{n=0}^{N} C_{n} .
\end{aligned}
$$

Using Eqs. (A2), we find that the expression under the integral in the vicinity of the points $p= \pm i \infty$ simplifies to

$$
\frac{\bar{W}(p) e^{s p} \prod_{n=0}^{N}\left(k_{n}^{2}+p^{2}\right)}{p \sum_{n=0}^{N}\left\{C_{n} \prod_{m \neq n}\left(k_{m}^{2}+p^{2}\right)\right\}} \underset{p \rightarrow \pm i \infty}{\longrightarrow} \frac{p \bar{W}(p) e^{s p}}{\sum_{n=0}^{N} C_{n}} .
$$

We recall from Eq. (11) that

$$
\mathcal{L}\left[W^{\prime}(s)\right]=p \bar{W}(p)-W(0),
$$

and assuming $W(0)=0$ we arrive at

$$
\begin{aligned}
\rho_{z}(s)= & \frac{W^{\prime}(s)}{\sum_{n=0}^{N} C_{n}}+\frac{1}{2 \pi i} \int_{\kappa-i \infty}^{\kappa+i \infty} \\
& \times\left[\frac{\bar{W}(p) e^{s p} \prod_{n=0}^{N}\left(k_{n}^{2}+p^{2}\right)}{p \sum_{n=0}^{N}\left\{C_{n} \prod_{m \neq n}\left(k_{m}^{2}+p^{2}\right)\right\}}-\frac{p \bar{W}(p) e^{s p}}{\sum_{n=0}^{N} C_{n}}\right] d p .
\end{aligned}
$$

The integral in the second term does not have an essential singularity anymore as it is canceled out.

To evaluate the integral in the Eq. (A1) first we apply convolution theorem for the Laplace transformation [17] in a form

$$
\mathcal{L}\left[W * K_{f}\right]=\bar{W}(p) \bar{K}_{f}(p)
$$

with $\bar{K}_{f}(p)$ given by

$$
\bar{K}_{f}(p)=\frac{\prod_{n=0}^{N}\left(k_{n}^{2}+p^{2}\right)}{p \sum_{n=0}^{N}\left\{C_{n} \prod_{m \neq n}\left(k_{m}^{2}+p^{2}\right)\right\}}-\frac{p}{\sum_{n=0}^{N} C_{n}} .
$$

This allows one to rewrite Eq. (A5) as

$$
\rho_{z}(s)=\frac{W^{\prime}(s)}{\sum_{n=0}^{N} C_{n}}+W(s) * K_{f}(s) .
$$

The next step is the evaluation of the integral $K(s)=$ $\mathcal{L}^{-1}[\bar{K}(p)]$

$$
\begin{aligned}
K_{f}(s)= & \frac{1}{2 \pi i} \int_{\kappa-i \infty}^{\kappa+i \infty} \\
& \times\left[\frac{e^{s p} \prod_{n=0}^{N}\left(k_{n}^{2}+p^{2}\right)}{p \sum_{n=0}^{N}\left\{C_{n} \prod_{m \neq n}\left(k_{m}^{2}+p^{2}\right)\right\}}-\frac{p e^{s p}}{\sum_{n=0}^{N} C_{n}}\right] d p .
\end{aligned}
$$

The integral in Eq. (A9) does not have any singularities in the left half plane (Fig. 6) except for the poles that are defined by the polynomial in the denominator of the first term. The corresponding equation for the poles reads

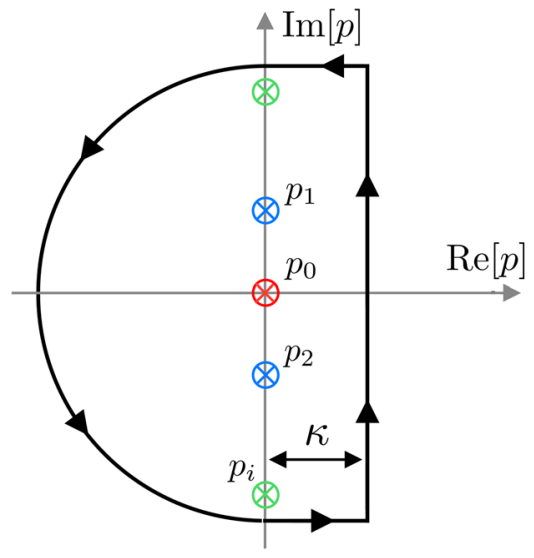

FIG. 6. Schematics of the integration contour for the inverse Laplace transformation.

$$
p \sum_{n=0}^{N}\left\{C_{n} \prod_{m \neq n}\left(k_{m}^{2}+p^{2}\right)\right\}=0 .
$$

Therefore the integral in Eq. (A9) could be calculated using the residue theorem $[17,18]$. It is apparent that the second term under the integral in Eq. (A9) does not contribute to these residues, thus

$$
K_{f}(s)=\sum_{p_{i}} \operatorname{Res}\left[\frac{e^{s p} \prod_{n=0}^{N}\left(k_{n}^{2}+p^{2}\right)}{p \sum_{n=0}^{N}\left\{C_{n} \prod_{m \neq n}\left(k_{m}^{2}+p^{2}\right)\right\}}\right] .
$$

We notice that the pole at $p=0$ could be always evaluated explicitly and reads

$$
\operatorname{Res}(p=0)=\frac{\prod_{n=0}^{N} k_{n}^{2}}{\sum_{n=0}^{N}\left\{C_{n} \prod_{m \neq n} k_{m}^{2}\right\}} .
$$

With Eq. (A11) and Eq. (A8) accounting for Eq. (A12) we finally arrive at

$$
\begin{aligned}
\rho_{z}(s)= & \frac{W^{\prime}(s)}{\sum_{n=0}^{N} C_{n}}+\frac{\prod_{n=0}^{N} k_{n}^{2}}{\sum_{n=0}^{N}\left\{C_{n} \prod_{m \neq n} k_{m}^{2}\right\}} \int_{0}^{s} W(\tilde{s}) d \tilde{s} \\
& +\int_{0}^{s} K(s-\tilde{s}) W(\tilde{s}) d \tilde{s} .
\end{aligned}
$$

Here $K(s)$ denotes the sum of residues that are defined by the roots of Eq. (A10) except for the trivial root $p=0$ :

$K(s)=\sum_{p_{i} \neq 0} \operatorname{Res}\left[\frac{e^{s p} \prod_{n=0}^{N}\left(k_{n}^{2}+p^{2}\right)}{p \sum_{n=0}^{N}\left\{C_{n} \prod_{m \neq n}\left(k_{m}^{2}+p^{2}\right)\right\}}\right]$.

[1] B. D. O'Shea, G. Andonian, S. K. Barber, K. L. Fitzmorris, S. Hakimi, J. Harrison, P. D. Hoang, M. J. Hogan, B. Naranjo, O. B. Williams, V. Yakimenko, and J. B. 
Rosenzweig, Observation of acceleration and deceleration in gigaelectron-volt-per-metre gradient dielectric wakefield accelerators, Nat. Commun. 7, 12763 (2016).

[2] M. Litos et al., High-efficiency acceleration of an electron beam in a plasma wakefield accelerator, Nature (London) 515, 92 (2014).

[3] K. L. F. Bane, P. Chen, and P. Wilson, On collinear wake field acceleration, Report No. SLAC-PUB, 1985, pp. 1-3.

[4] R. J. England, J. B. Rosenzweig, and G. Travish, Generation and Measurement of Relativistic Electron Bunches Characterized by a Linearly Ramped Current Profile, Phys. Rev. Lett. 100, 214802 (2008).

[5] G. Berden, S. P. Jamison, A. M. MacLeod, W. A. Gillespie, B. Redlich, and A.F. G. van der Meer, Electro-optic Technique with Improved Time Resolution for Real-time, Nondestructive, Single-shot Measurements of Femtosecond Electron Bunch Profiles, Phys. Rev. Lett. 93, 114802 (2004).

[6] U. Happek, A. J. Sievers, and E. B. Blum, Observation of Coherent Transition Radiation, Phys. Rev. Lett. 67, 2962 (1991).

[7] G. Andonian, A. Cook, M. Dunning, E. Hemsing, G. Marcus, A. Murokh, S. Reiche, D. Schiller, J. B. Rosenzweig, M. Babzien, K. Kusche, and V. Yakimenko, Observation of coherent terahertz edge radiation from compressed electron beams, Phys. Rev. Accel. Beams 12, 030701 (2009).

[8] H. L. Andrews, F. B. Taheri, J. Barros, R. Bartolini, V. Bharadwaj, C. Clarke, N. Delerue, G. Doucas, N. Fuster-Martinez, M. Vieille-Grosjean, I. V. Konoplev, M. Labat, S. Le Corre, C. Perry, A. Reichold, and S. Stevenson, Reconstruction of the time profile of 20.35 $\mathrm{GeV}$, subpicosecond long electron bunches by means of coherent Smith-Purcell radiation, Phys. Rev. Accel. Beams 17, 052802 (2014).

[9] W. K. H. Panofsky and W. A. Wenzel, Some considerations concerning the transverse deflection of charged particles in radiofrequency fields, Rev. Sci. Instrum. 27, 967 (1956).

[10] Q. Gao, J. Shi, H. Chen, G. Ha, J. G. Power, M. Conde, and W. Gai, Single-shot wakefield measurement system, Phys. Rev. Accel. Beams 21, 062801 (2018).

[11] L. Zhao, T. Jiang, C. Lu, R. Wang, Z. Wang, P. Zhu, Y. Shi, W. Song, X. Zhu, C. Jing, S. Antipov, D. Xiang, and J. Zhang, Few-femtosecond electron beam with terahertzfrequency wakefield-driven compression, Phys. Rev. Accel. Beams 21, 082801 (2018).

[12] G. Andonian, S. Barber, F. H. O'Shea, M. Fedurin, K. Kusche, C. Swinson, and J. B. Rosenzweig, Generation of Ramped Current Profiles in Relativistic Electron Beams using Wakefields in Dielectric Structures, Phys. Rev. Lett. 118, 054802 (2017).

[13] S. Antipov, S. Baturin, C. Jing, M. Fedurin, A. Kanareykin, C. Swinson, P. Schoessow, W. Gai, and A. Zholents, Experimental Demonstration of Energy-Chirp Compensation by a Tunable Dielectric-based Structure, Phys. Rev. Lett. 112, 114801 (2014).

[14] P. Emma, M. Venturini, K. L. F. Bane, G. Stupakov, H.-S. Kang, M. S. Chae, J. Hong, C.-K. Min, H. Yang, T. Ha, W. W. Lee, C. D. Park, S. J. Park, and I. S. Ko, Experimental Demonstration of Energy-Chirp Control in
Relativistic Electron Bunches Using a Corrugated Pipe, Phys. Rev. Lett. 112, 034801 (2014).

[15] A. Novokhatski, A. Brachmann, M. Dal Forno, V. A. Dolgashev, A. S. Fisher, M. W. Guetg, Z. Huang, R. H. Iverson, P. Krejcik, A. A. Lutman, T. J. Maxwell, and J. Zemella, RadiaBeam/SLAC dechirper as a passive deflector, in 7th International Particle Accelerator Conference (JACoW, Geneva, 2016), p. MOPOW046.

[16] A. Chao, Physics of Collective Beam Instabilities in High Energy Accelerators (Wiley, New York, 1993).

[17] M. A. Lavrentiev and B. V. Shabat, Methods of Complex Function Theory (Nauka, Moskow, 1987).

[18] R. A. Silverman, Introductory Complex Analysis (Dover Publications, Inc., New York, 1972).

[19] R. Roussel, G. Andonian, W. Lynn, K. Sanwalka, R. Robles, C. Hansel, A. Deng, G. Lawler, J. B. Rosenzweig, G. Ha, J. Seok, J. G. Power, M. Conde, E. Wisniewski, D. S. Doran, and C. E. Whiteford, Single Shot Characterization of High Transformer Ratio Wakefields in Nonlinear Plasma Acceleration, Phys. Rev. Lett. 124, 044802 (2020).

[20] Q. Gao, G. Ha, C. Jing, S. P. Antipov, J. G. Power, M. Conde, W. Gai, H. Chen, J. Shi, E. E. Wisniewski, D. S. Doran, W. Liu, C. E. Whiteford, A. Zholents, P. Piot, and S. S. Baturin, Observation of High Transformer Ratio of Shaped Bunch Generated by an Emittance-Exchange Beam Line, Phys. Rev. Lett. 120, 114801 (2018).

[21] G. Andonian, O. Williams, S. Barber, D. Bruhwiler, P. Favier, M. Fedurin, K. Fitzmorris, A. Fukasawa, P. Hoang, K. Kusche, B. Naranjo, B. O'Shea, P. Stoltz, C. Swinson, A. Valloni, and J.B. Rosenzweig, Planar-DielectricWakefield Accelerator Structure Using Bragg-reflector Boundaries, Phys. Rev. Lett. 113, 264801 (2014).

[22] P. D. Hoang, G. Andonian, I. Gadjev, B. Naranjo, Y. Sakai, N. Sudar, O. Williams, M. Fedurin, K. Kusche, C. Swinson, P. Zhang, and J. B. Rosenzweig, Experimental Characterization of Electron-Beam-driven Wakefield Modes in a Dielectric-Woodpile Cartesian Symmetric Structure, Phys. Rev. Lett. 120, 164801 (2018).

[23] S. S. Baturin, G. Andonian, and J. B. Rosenzweig, Analytical treatment of the wakefields driven by transversely shaped beams in a planar slow-wave structure, Phys. Rev. Accel. Beams 21, 121302 (2018).

[24] F. Lemery and P. Piot, Tailored electron bunches with smooth current profiles for enhanced transformer ratios in beam-driven acceleration, Phys. Rev. Accel. Beams 18, 081301 (2015).

[25] S. S. Baturin and A. Zholents, Upper limit for the accelerating gradient in the collinear wakefield accelerator as a function of the transformer ratio, Phys. Rev. Accel. Beams 20, 061302 (2017).

[26] C. Li, W. Gai, C. Jing, J. G. Power, C. X. Tang, and A. Zholents, High gradient limits due to single bunch beam breakup in a collinear dielectric wakefield accelerator, Phys. Rev. Accel. Beams 17, 091302 (2014).

[27] S. S. Baturin and A. Zholents, Stability condition for the drive bunch in a collinear wakefield accelerator, Phys. Rev. Accel. Beams 21, 031301 (2018).

[28] P. Piot, Y.-E Sun, J. G. Power, and M. Rihaoui, Generation of relativistic electron bunches with arbitrary current 
distribution via transverse-to-longitudinal phase space exchange, Phys. Rev. Accel. Beams 14, 022801 (2011).

[29] W. Lu, C. Huang, M. Zhou, M. Tzoufras, F. S. Tsung, W. B. Mori, and T. Katsouleas, A nonlinear theory for multidimensional relativistic plasma wave wakefields, Phys. Plasmas 13, 056709 (2006).

[30] J. B. Rosenzweig, Trapping, thermal effects, and wave breaking in the nonlinear plasma wake-field accelerator, Phys. Rev. A 38, 3634 (1988).

[31] M. J. Hogan, T. O. Raubenheimer, A. Seryi, P. Muggli, T. Katsouleas, C. Huang, W. Lu, W. An, K. A. Marsh, W. B. Mori, C. E. Clayton, and C. Joshi, Plasma wakefield acceleration experiments at FACET, New J. Phys. 12, 055030 (2010).

[32] W. Lu, C. Huang, M. M. Zhou, W. B. Mori, and T. Katsouleas, Limits of linear plasma wakefield theory for electron or positron beams, Phys. Plasmas 12, 063101 (2005).

[33] D. P. Grote, A. Friedman, J. L. Vay, and I. Haber, The warp code: Modeling high intensity ion beams, AIP Conf. Proc. 749, 55 (2005).

[34] R. Roussel, G. Andonian, C. Hansel, G. Lawler, W. Lynn, N. Majernik, R. Robles, K. Sanwalka, E. Wisniewski, and J. Rosenzweig, Externally heated hollow cathode arc plasma source for experiments in plasma wakefield acceleration, Instruments 3, 48 (2019).

[35] R. Roussel, G. Andonian, W. Lynn, J. Rosenzweig, J. Power, M. Conde, S. Doran, G. Ha, J. Seok, E. Wisniewski, and C. Whiteford, Transformer ratio measurements from ramped beams in the plasma blowout regime using emittance exchange, in Proceedings of IPAC2019 (JACoW, Geneva, 2019), p. 3778.

[36] N. Barov and J. B. Rosenzweig, Propagation of short electron pulses in underdense plasmas, Phys. Rev. E 49, 4407 (1994).
[37] G. R. Lynch and O. I. Dahl, Approximations to multiple coulomb scattering, Nucl. Instrum. Methods Phys. Res., Sect. B, 58, 6 (1991).

[38] G. Andonian, D. Stratakis, M. Babzien, S. Barber, M. Fedurin, E. Hemsing, K. Kusche, P. Muggli, B. O'Shea, X. Wei, O. Williams, V. Yakimenko, and J. B. Rosenzweig, Dielectric Wakefield Acceleration of a Relativistic Electron Beam in a Slab-Symmetric Dielectric Lined Waveguide, Phys. Rev. Lett. 108, 244801 (2012).

[39] B. Podobedov and G. Stupakov, Point-charge wakefield calculations from finite length bunch wake potentials, Phys. Rev. Accel. Beams 16, 024401 (2013).

[40] S. S. Baturin, I. L. Sheinman, A. M. Altmark, and A. D. Kanareykin, Transverse operator method for wakefields in a rectangular dielectric loaded accelerating structure, Phys. Rev. Accel. Beams 16, 051302 (2013).

[41] A. Tremaine, J. Rosenzweig, and P. Schoessow, Electromagnetic wake fields and beam stability in slabsymmetric dielectric structures, Phys. Rev. E 56, 7204 (1997).

[42] D. Mihalcea, P. Piot, and P. Stoltz, Three-dimensional analysis of wakefields generated by flat electron beams in planar dielectric-loaded structures, Phys. Rev. Accel. Beams 15, 081304 (2012).

[43] E. Adli et al., Acceleration of electrons in the plasma wakefield of a proton bunch, Nature (London) 561, 363 (2018).

[44] V. Yakimenko, L. Alsberg, E. Bong, G. Bouchard, C. Clarke, C. Emma, S. Green, C. Hast, M. J. Hogan, J. Seabury, N. Lipkowitz, B. O’Shea, D. Storey, G. White, and G. Yocky, Facet-II facility for advanced accelerator experimental tests, Phys. Rev. Accel. Beams 22, 101301 (2019). 